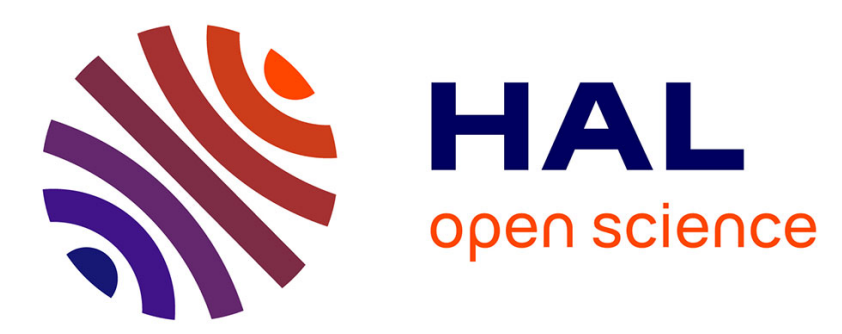

\title{
Topographically induced internal solitary waves in a pycnocline: Secondary generation and selection criteria
}

\author{
Yvan Dossmann, F. Auclair, A. Paci
}

\section{To cite this version:}

Yvan Dossmann, F. Auclair, A. Paci. Topographically induced internal solitary waves in a pycnocline: Secondary generation and selection criteria. Physics of Fluids, 2013, 25, pp.86603 - 86603. 10.1063/1.4817373 . hal-01753326

\section{HAL Id: hal-01753326 \\ https://hal.univ-lorraine.fr/hal-01753326}

Submitted on 17 May 2018

HAL is a multi-disciplinary open access archive for the deposit and dissemination of scientific research documents, whether they are published or not. The documents may come from teaching and research institutions in France or abroad, or from public or private research centers.
L'archive ouverte pluridisciplinaire HAL, est destinée au dépôt et à la diffusion de documents scientifiques de niveau recherche, publiés ou non, émanant des établissements d'enseignement et de recherche français ou étrangers, des laboratoires publics ou privés. 


\title{
Topographically induced internal solitary waves in a pycnocline: Secondary generation and selection criteria
}

\author{
Y. Dossmann, ${ }^{1,2,3, a)}$ F. Auclair, ${ }^{2}$ and A. Paci ${ }^{3}$ \\ ${ }^{1}$ Research School of Earth Sciences, The Australian National University, \\ Canberra 0200, Australia \\ ${ }^{2}$ Laboratoire d'Aérologie (UMR 5560 CNRS \& UPS Toulouse III), 14 avenue Edouard Belin, \\ 31400 Toulouse, France \\ ${ }^{3}$ CNRM-GAME/GMEI (UMR3589 Météo-France \& CNRS), 42 Avenue Gaspard Coriolis, \\ 31057 Toulouse Cedex 01, France
}

(Received 8 January 2013; accepted 19 July 2013; published online 16 August 2013)

Geophysical flows support the propagation of stable nonlinear internal waves (internal solitary waves or ISWs) with complex generation mechanisms. At least two regimes of ISWs generation in the pycnocline, both involving the interaction between a tidal flow and the bottom topography, are known in the ocean. They can either be directly induced above topographies (primary generation) or by a topographic internal wave beam impinging on the pycnocline. This "secondary generation" process is the subject-matter of the present study. The present work relies on direct numerical simulations of an academic configuration inspired by oceanic observations. It aims at describing the different steps involved in the secondary generation process. To mimic the oceanic case, the internal wave beam is emitted from the topography at the bottom of the flow. First, the linear scattering of the internal wave beam at the pycnocline is studied in a linear configuration. Increasing the forcing amplitude leads to the generation of steep isopycnal troughs in the pycnocline, at the locations of the internal wave beam impacts. The dynamics of these troughs is studied in details, which permits to associate them with propagating ISW2s that emerge from the second normal mode. Finally, the evolution of the structure of normal modes 2 and 3 with respect to the pycnocline strength, as well as the role played by the topography, is analyzed. This study is a step to complete and unify previous independent analytical studies of the secondary generation process. ( 2013 AIP Publishing LLC. [http://dx.doi.org/10.1063/1.4817373]

\section{INTRODUCTION}

Internal solitary waves (ISWs) are commonly observed in atmospheric (Tsai, Kanamori, and Artru, ${ }^{1}$ Rottman and Grimshaw ${ }^{2}$ ) and oceanic (Apel et al., ${ }^{3}$ Halpern, ${ }^{4}$ New and Pingree ${ }^{5}$ ) flows. In these geophysical fluids, strongly stratified regions can be viewed as a horizontal waveguide for the propagation of ISWs. Their stability arises from the balance between nonlinear effects on one hand and dispersion induced by nonhydrostatic and rotational effects on the other hand (Helfrich and Melville ${ }^{6}$ ).

In the ocean, ISWs propagate at the ocean pycnocline (the bottom of the oceanic mixed layer) in a quasi interfacial manner. In the course of their propagation, the strong vertical displacements that they induce can modify the dynamical and biological properties of the mixed layer (Kantha and Clayson, ${ }^{7}$ Lai et al. ${ }^{8}$ ). To quantify the impact of these waves, it is necessary to understand the mechanism leading to their generation.

At least two regimes of ISWs generation by the interaction between a tidal flow and the bottom topography are known in the ocean. It has been commonly observed, for example, in the Sulu Sea

a)yvan.dossmann@anu.edu.au 
and in the Celtic Sea (Apel et al., ${ }^{3}$ Pingree and Mardell ${ }^{9}$ ), that the direct interaction between the barotropic tide and a steep topography can generate ISWs propagating the ocean pycnocline. The term "primary generation" and ISW1s are adopted to refer to this process and the ISWs it generates, in accordance with a previous numerical study (Dossmann, Auclair, and Paci ${ }^{10}$ ). In the latter study, an important topographic control of the primary generation process was extracted.

An altogether different ISW generation process has been convincingly described by New and Pingree, ${ }^{5,11}$ using observational measurements in the Bay of Biscay. In this case, ISWs are generated $150 \mathrm{~km}$ away from the shelf break by the impingement on the pycnocline of a topographic internal wave beam. The term "local generation" was coined by New and Pingree ${ }^{5}$ to refer to this mechanism. We adopt the terms "secondary generation" and ISW2s in the present article as opposed to the "primary generation" process and ISW1s described in Dossmann, Auclair, and Paci. ${ }^{10}$

New and Da Silva ${ }^{12}$ analyzed synthetic aperture radar (SAR) data, to observe ISW1s emitted at the shelf break, and ISW2s emitted approximately $200 \mathrm{~km}$ away from any topography in the northern and central Bay of Biscay. They were able to isolate the two generation processes as both sets of ISWs were seen to decay after travelling $100 \mathrm{~km}$. Seamounts and ridges can also be efficient ISW2s generators, as observed by Azevedo, da Silva, and $\mathrm{New}^{13}$ on the two sides of the Ortegal Promontory.

The complexity of the secondary generation process raises the need to extract simple parameters to describe it. Delisi and Orlanski ${ }^{14}$ performed laboratory experiments to investigate the interaction between an internal wave beam generated in a stratified layer and the pycnocline. They showed with a linear theoretical approach that the pycnocline strength (comparing the magnitude of the density jump in the pycnocline with the lower layer stratification) partly controls the amplitude of the vertical motions induced in the pycnocline. This result was extended with a weakly nonlinear approach by Thorpe ${ }^{15}$ with an application to the Bay of Biscay.

Gerkema ${ }^{16}$ analytically solved a model of linear internal-tide generation over an infinitesimal topography, with a zero-thickness pycnocline, using a normal mode approach. In the regime of a moderate pycnocline strength, a substantial energy transfer from the internal wave beam to the interfacial wave in the pycnocline occurs via a linear scattering process. By numerically solving a weakly nonlinear nonhydrostatic model, Gerkema ${ }^{16}$ showed that large depressions are induced in the pycnocline in this regime. The depressions evolve into propagating ISW2s when nonlinear and dispersive effects reach a balance.

Gerkema ${ }^{17}$ extended the description of the previous study to a multimodal approach applied to moderate (Bay of Biscay) and strong (Sulu Sea) pycnocline strength. In the former regime, substantial energy transfers between normal modes leading to a downward leaking of energy from the propagating ISW2s to internal waves propagating in the lower layer were observed. At the contrary, ISW1s generated in the Sulu Sea propagate in a unimodal, quasi-interfacial manner, what explains their remarkable stability with respect to ISW2s. In order to describe more realistic configurations, the multi-modal approach was used in a weakly nonlinear nonhydrostatic model considering topographies with large extent in front of the beam horizontal wavelength (Maugé and Gerkema ${ }^{18}$ ). Model solving with realistic topography, stratification, and tidal forcing from the Bay of Biscay showed a good correspondence with fully numerical model outputs.

Akylas et al. ${ }^{19}$ used a theoretical, weakly nonlinear model of the interaction between an internal wave beam of prescribed shape propagating in the lower, stratified layer and the pycnocline of zero thickness. They showed that the matching between the interfacial wavelength on one hand, and the horizontal wavelength in the internal wave beam on the other hand, leads to the largest vertical motions in the pycnocline. Note that it corresponds to a phase speed matching as the frequencies are identical in the stratified layer and the pycnocline before nonlinear effects come into play. Although this study relies on a different physical approach from the one of Gerkema, ${ }^{16}$ the overall ISW2 dynamics are similar.

Direct numerical simulations performed by Grisouard et al. ${ }^{20}$ focused on the secondary generation process for a pycnocline of finite thickness by using an internal wave beam shaped with an analytical function in the lower layer. The authors argue that mode- $n$ ISW2s are efficiently generated when the horizontal phase speed of the internal wave beam matches the phase speed of a normal mode $n$ trapped in the pycnocline. Their application to the Bay of Biscay, using a nested-grid 
approach to model topographic internal wave beams, revealed that the interaction between ISW2s and a linear interfacial wave may impose the spatial distance between consecutive ISW2s trains (Grisouard and Staquet ${ }^{21}$ ).

In the present study, the secondary generation of ISW2s over a ridge is presented in an academic configuration at the laboratory scale using direct numerical simulations. To provide a more complete insight of the secondary generation process, we aim at directly modelling the three main stages involved:

1. the generation of an internal wave beam over the topography,

2. the linear scattering of the internal wave beam impinging on the pycnocline,

3. the evolution of the induced strong interfacial displacement into a propagating ISW2.

The periodic oscillation of a Gaussian ridge in a linearly stratified bottom layer generates upgoing and downgoing internal wave beams, whose interaction with the pycnocline is described. In particular, the beam shape is controlled by the ridge shape, the stratification and the forcing, enabling a step further towards a realistic configuration. The linear and nonlinear regimes are studied in order to observe the linear scattering of internal wave beams on the pycnocline, and the subsequent secondary generation of ISW2s. A novel description of the evolution of the normal modes structure with the pycnocline strength is proposed to extract a general criterion, valid for all modes, controlling the secondary generation process. The role played by the topography shape is also tackled. Relying on this description, we propose to link between previous independent approaches of the secondary generation process (Gerkema, ${ }^{16}$ Akylas et al. ${ }^{19}$ Grisouard and Staquet ${ }^{21}$ ).

In Sec. II, we present the configuration adopted for studying the secondary generation process. Section III describes the internal wave dynamics in the case of a millimetric forcing amplitude, with a particular focus on the linear scattering process. In Sec. IV, we describe the secondary generation of ISW at a higher forcing amplitude. A normal mode approach is used in Sec. V to extract a general selection criterion for this process. In Sec. VI, some hints to unify different descriptions of the secondary generation process are provided, by highlighting the role played by the topography shape. Conclusions are drawn in Sec. VII.

\section{NUMERICAL AND PHYSICAL CONFIGURATION}

\section{A. General features of the numerical model}

Simulations are performed using the nonhydrostatic version of the regional oceanic circulation model Symphonie-NH described in Auclair et al. ${ }^{22}$ Symphonie-NH solves the equations of motion under the Boussinesq approximation, with a linear equation of state in the present case, onto the Arakawa-C grid using s-coordinates. Density depends on salinity, while temperature is set to a constant value. Time-splitting between barotropic and baroclinic motions is performed for the temporal discretization, based on a centered leap-frog scheme, in order to reduce computational costs.

A bidimensional version of the model in a vertical plane is used, with no transverse diffusive fluxes and no transverse motions allowed. Free surface boundary conditions and no-slip condition at the bottom of the domain are used. A moving floor implemented in the model allows a forcing by the oscillation of the topography, in order to facilitate comparisons with laboratory experiments. Simulations are carried out on 32 or $64 \mathrm{CPU}$, by splitting the domain horizontally with the Message Passing Interface (MPI) library.

\section{B. Configuration}

Direct numerical simulations of internal wave generation at short scales permitting high resolution and easy comparisons with laboratory experiments are carried out. The horizontal length scale and resolution are $L=16 \mathrm{~m}$ and $d x=1 \mathrm{~mm}$. The fluid depth is $H=40 \mathrm{~cm}$, with 100 regularly spaced s-levels in the vertical direction, corresponding to an average vertical resolution of $4 \mathrm{~mm}$. Diffusion coefficients for salinity and velocities are set to their molecular values recalled in Table I. 
TABLE I. Physical and numerical parameters.

\begin{tabular}{lcc}
\hline \hline Designation & Name & Value or range \\
\hline Physical configuration & & \\
Total fluid depth & $L$ & $40 \mathrm{~cm}$ \\
Domain length & $h_{1}$ & $16 \mathrm{~m}$ \\
Upper layer depth & $\rho_{1}$ & $1.5 \mathrm{~cm}$ \\
Upper layer density & $\delta_{p}$ & $1000 \mathrm{~kg} / \mathrm{m}^{3}$ \\
Pycnocline thickness & $\Delta \rho$ & $1 \mathrm{~cm}$ \\
Density jump & $h_{2}$ & $80 \mathrm{~kg} / \mathrm{m}^{3}$ \\
Bottom layer depth & $N_{0}$ & $37.5 \mathrm{~cm}$ \\
Brunt-Väisälä frequency in the bottom layer & $h_{0}$ & $2 \mathrm{rad} / \mathrm{s}$ \\
Ridge height & $l$ & $25 \mathrm{~cm}$ \\
Ridge e-folding width & $A$ & $10 \mathrm{~cm}$ \\
Forcing amplitude & $a=A / l$ & {$[1,20] \mathrm{mm}$} \\
Tidal excursion & $T$ & {$[0.01,0.2]$} \\
Forcing period & $\omega=2 \pi / T$ & $5 \mathrm{~s}$ \\
Forcing frequency & $\sqrt{g \Delta \rho / \rho_{1} h_{1}}$ & $1.26 \mathrm{rad} / \mathrm{s}$ \\
Linear longwave speed & $\theta$ & $0.13 \mathrm{~m} / \mathrm{s}$ \\
Internal wave beam theoretical angle in the bottom layer & $\gamma$ & $39^{\circ}$ \\
Gerkema (2001) pycnocline strength & $\alpha$ & 0.15 \\
Akylas (2007) pycnocline strength & $v$ & 0.9 \\
Kinematic viscosity & $c_{S}$ & $10^{-6} \mathrm{~m}^{2} / \mathrm{s}$ \\
Salinity diffusivity & & $10^{-9} \mathrm{~m} / \mathrm{s}$ \\
Numerical parameters & $d x$ & $1 \mathrm{~mm}$ \\
Horizontal resolution & $d z$ & $4 \mathrm{~mm}$ \\
Average vertical resolution & $t_{e}$ & $3.21 \times 10^{-4} \mathrm{~s}$ \\
External time step & $t_{i}$ & $1.28 \times 10^{-3} \mathrm{~s}$ \\
Internal time step & &
\end{tabular}

Simulations are initialized with a continuous density profile $\bar{\rho}(z)$ typical of oceanic profiles. It consists of a homogeneous upper layer of depth $h_{1}=1.5 \mathrm{~cm}$ and density $\rho_{1}=1000 \mathrm{~kg} / \mathrm{m}^{3}$, representing the oceanic mixing layer, but at the ocean scale. The linearly stratified bottom layer of depth $h_{2}=37.5 \mathrm{~cm}$ and constant Brunt-Väisälä frequency $N_{0}=2 \mathrm{rad} / \mathrm{s}$ represents the abyssal ocean. A $1 \mathrm{~cm}$ wide pycnocline $\left(\delta_{p}=1 \mathrm{~cm}\right)$ with a density jump $\Delta \rho=80 \mathrm{~kg} / \mathrm{m}^{3}$ separates the two layers. The pycnocline center is at a position $z=-h_{p}=-h_{1}-\delta_{p} / 2=-2 \mathrm{~cm}$. The corresponding Brunt-Väisälä frequency is the same as the one used in Grisouard et al.: ${ }^{20}$

$$
N^{2}(z)=\frac{2}{\sqrt{\pi}} g \frac{\Delta \rho}{\delta_{p}} \exp \left[-\left(\frac{z+h_{p}}{\delta / 2}\right)^{2}\right]+ \begin{cases}N_{0}^{2} & \text { for }-H \leq z<-h_{p} \\ 0 & \text { for }-h_{p} \leq z \leq 0 .\end{cases}
$$

To facilitate comparisons with laboratory experiments, simulations are performed with a "moving bottom" version of the model. The forcing is consequently performed through the oscillation of a Gaussian ridge whose shape is given by

$$
h(x)=h_{0} \exp \left(-\left(\frac{x}{l}\right)^{2}\right)
$$

where $h_{0}$ and $l$ are the height and the e-folding width of the ridge. The topography is referred to as supercritical (respectively, subcritical) in a configuration where the slope of the characteristics is smaller (respectively, greater) than the maximum slope of the topography. The ridge displacement is given by $x_{m}(t)=A \cos (2 \pi t / T)$, where $x_{m}(t)$ is the horizontal position of the center of the ridge, $(A, T)$ are the forcing amplitude and period.

The internal wave beam angles $\theta$ with respect to the horizontal are always comprised between $0^{\circ}$ and $90^{\circ}$. It is specified whether the beam propagates upwards or downwards. The domain origin 


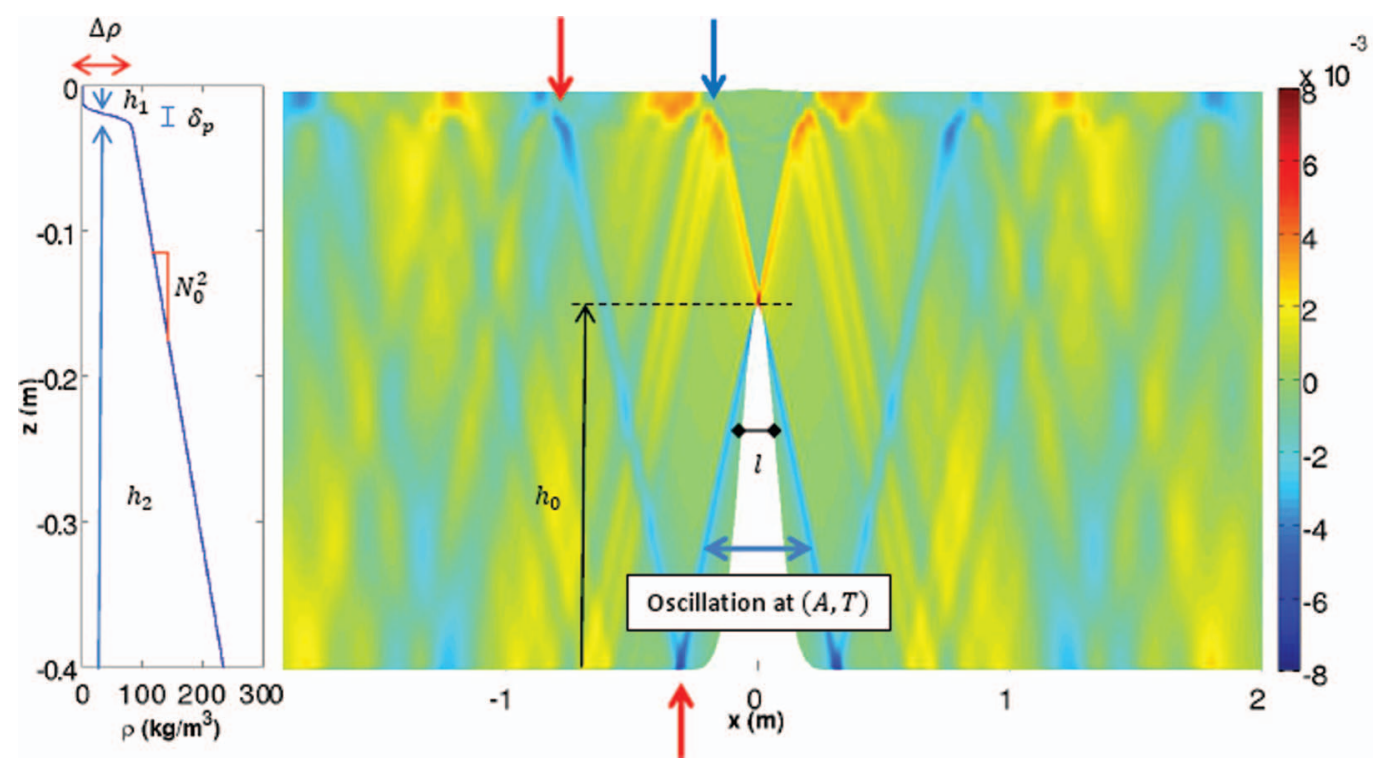

FIG. 1. Horizontal velocity field $u(\mathrm{~m} / \mathrm{s})$ in $\operatorname{Sim} 9$ at $t=14 \mathrm{~T}$, caused by the oscillation of the ridge at an amplitude $A=1 \mathrm{~mm}$ and period $T=5 \mathrm{~s}$. The ridge height and e-folding width are $h_{0}=25 \mathrm{~cm}$ and $l=10 \mathrm{~cm}$, respectively. Useful parameters and the reference density profile $\bar{\rho}(z)$ are recalled at the left of the figure. Blue and red arrows indicate the locations where the internal wave beams scatter on the pycnocline.

is set at the average ridge horizontal position and at the free surface. The set-up parameters are recalled in Table I.

\section{LINEAR INTERNAL WAVE BEAM/PYCNOCLINE INTERACTION}

\section{A. Near field}

In order to study the structure of the linear internal wave field, a numerical simulation Sim9 with an amplitude forcing $A=1 \mathrm{~mm}$ is presented (Sim1 to $\operatorname{Sim} \mathbf{8}$ deal with the primary generation configuration, see Dossmann, Auclair, and $\mathrm{Paci}^{10}$ ). One can a priori expect that internal waves have a mostly linear behavior in $\operatorname{Sim} 9$ as the excursion parameter $a=A / l=0.01$ is much smaller than 1 . In fact, in a previous experimental study using a steeper ridge and similar forcing in amplitude and period, nonlinear effects were negligible in the internal wave field Dossmann et $_{\text {al }}{ }^{23}$

The horizontal velocity field $u(x, z, t=14 T)$ is shown in Figure 1 . As it is symmetrical with respect to $x=0$, we focus on the physical features at the left of the ridge with no loss of generality. One smooth, narrow, internal wave beam is emitted downwards over the ridge and propagates at a constant angle $\theta \approx 40$ to the bottom of the fluid. After a reflection at $x \approx-0.25 \mathrm{~m}$ (bottom red arrow) this internal wave beam crosses the whole lower layer at the same constant angle $\theta$ and eventually reaches the pycnocline at $x \approx-0.80 \mathrm{~m}$ (top red arrow). Another internal wave beam is emitted upwards and impacts the pycnocline at $x \approx-0.20 \mathrm{~m}$ (blue arrow). The internal wave beams reflected in the lower layer are (linearly) scattered, owing to the series of refraction/reflection occurring in the pycnocline, according to the results of Mathur and Peacock ${ }^{24}$ and Gerkema. ${ }^{16}$ In the upper layer where internal waves cannot propagate, patches of non propagating horizontal velocities with a $10 \mathrm{~cm}$ extent are observed. Overall, the internal wave field structure is strongly affected by the presence of the pycnocline.

Hence, one can infer that the present stratification corresponds to the moderate pycnocline regime described by Gerkema. ${ }^{16,17}$ The density jump in the pycnocline is weak enough to permit a substantial refraction of the internal wave beam in the pycnocline, yet it is strong enough for an important angle change to occur in the pycnocline, leading to an important linear scattering process. In fact, the parameter $\gamma$ is close to 0.16 , what corresponds to a moderate pycnocline in 

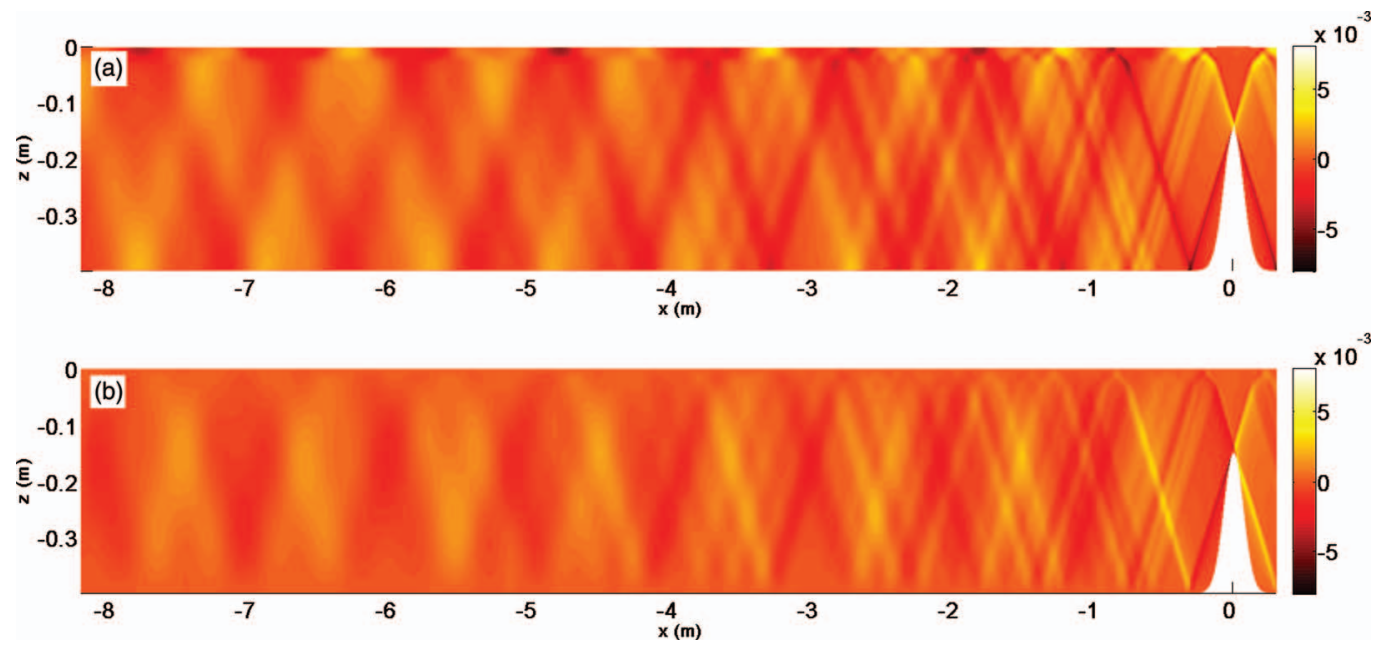

FIG. 2. (a) Horizontal $(u(\mathrm{~m} / \mathrm{s}))$ and (b) vertical $(w(\mathrm{~m} / \mathrm{s}))$ velocity fields at $t=22 \mathrm{~T}$, in $\operatorname{Sim9}$.

Gerkema. ${ }^{16}$ As a consequence, the internal wave beam is wider and exhibits more small scale features after the first reflection. Note that this linear scattering may impact the efficiency of the induced diapycnal mixing by weakening the amplitude of internal wave beams in the lower layer.

\section{B. Far field}

The horizontal and vertical velocity fields at $t=22 \mathrm{~T}$ are displayed in Figures 2(a) and 2(b), respectively. In both fields, consecutive reflections at the pycnocline provoke a progressive widening and an amplitude weakening in the internal wave beams. Only weak vertical velocities are observed in the mixing layer. This can be explained by the fact that the displacement anomalies of the internal waves propagating in the pycnocline are mainly horizontal. For this millimetric amplitude forcing, the pycnocline displacements induced by the internal wave beams impact hardly exceed $2-3 \mathrm{~mm}$ (not shown), and nonlinear processes are almost absent.

\section{SECONDARY GENERATION OF INTERNAL SOLITARY WAVES}

\section{A. Internal wave fields}

To study the secondary generation process in a configuration close to Sim9, it is necessary to allow vertical motions of larger amplitude in the pycnocline, permitting a possible balance between nonlinear and dispersive effects. For that purpose, the simulation Sim10, identical to Sim9 except for the forcing amplitude set 20 times larger, has been performed.

Velocity and density fields after $22 \mathrm{~T}$ are shown in Figure 3 . The internal wave beams propagating in the lower layer are overall wider than in the linear regime. They provoke local, steep isopycnal disformations of centimetric magnitude in the course of their propagation. Turbulent structures induced by the ridge oscillation are visible for $-2 \mathrm{~m} \leq x \leq 0 \mathrm{~m}$. When impinging on the pycnocline, internal wave beams are subject to the same linear scattering at the pycnocline as the one described previously. The initially upgoing internal wave beam undergoes an important energy loss after the first impact on the pycnocline, implying potential important energy transfers to propagating waves in the pycnocline. Narrow isopycnal troughs, directed towards the lower layer develop at $x \approx-0.80 \mathrm{~m}$, $x \approx-1.90 \mathrm{~m}, x \approx-3.60 \mathrm{~m}$ corresponding to the location of internal wave beams impacts. Their steepness indicates that nonlinear effects are probably at play. For $x \leq-4 \mathrm{~m}$, scattered internal wave beams induce smooth, harmonic isopycnal displacements of approximate magnitude $2 \mathrm{~cm}$ in the 


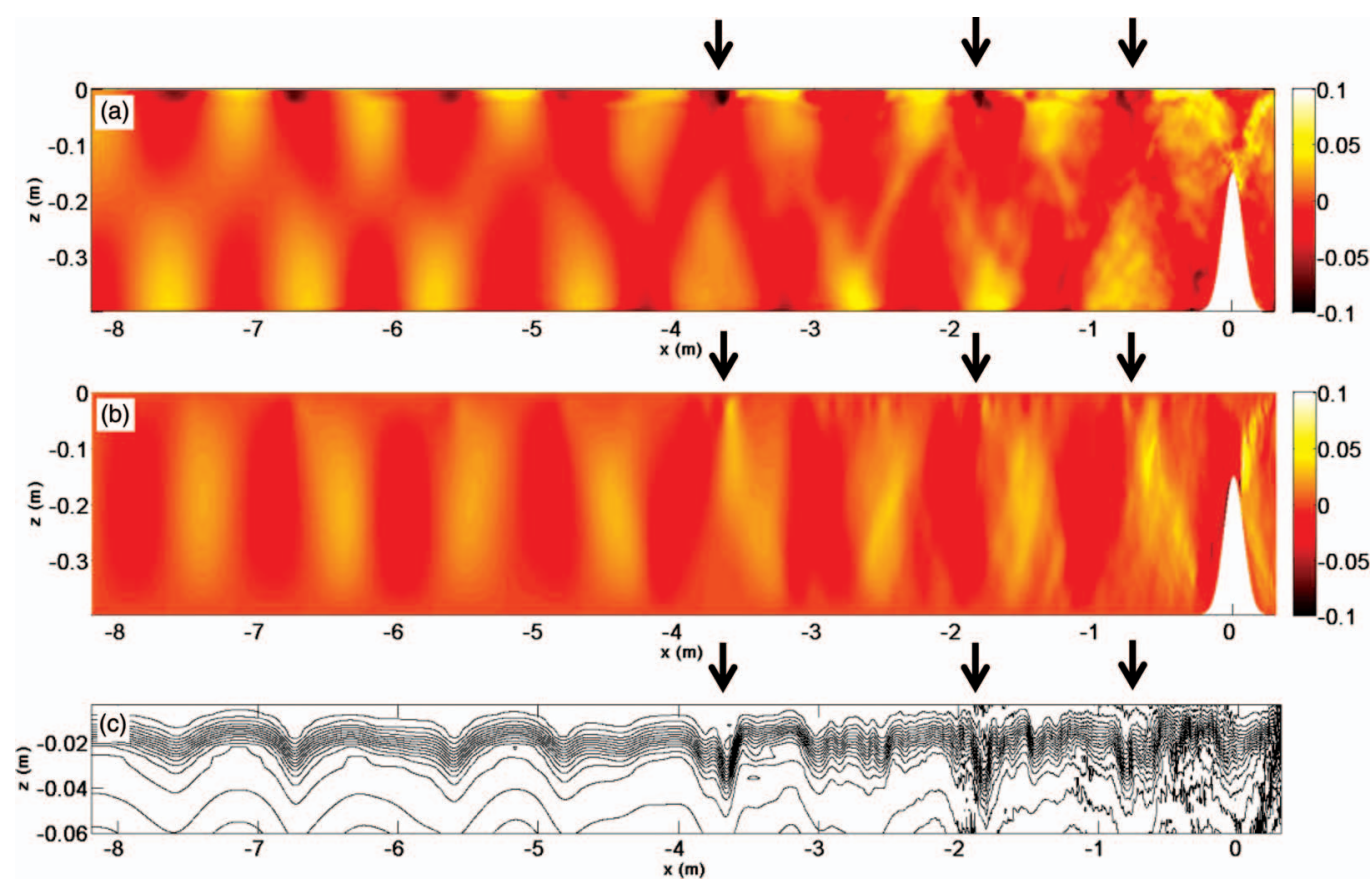

FIG. 3. (a) Horizontal velocity $(u(\mathrm{~m} / \mathrm{s}))$, (b) vertical velocity $(w(\mathrm{~m} / \mathrm{s}))$, and (c) density $\left(\rho\left(\mathrm{kg} / \mathrm{m}^{3}\right)\right)$ fields at $t=22 \mathrm{~T}$, in Sim10. Black arrows indicate the troughs induced by an internal wave beam impact on the pycnocline. Grey arrows show smaller isopycnal troughs in the pycnocline that have possibly radiated energy in the lower layer.

lower layer, while narrow, localized isopycnal troughs are observed at $x \approx-4.90 \mathrm{~m}, x \approx-5.60 \mathrm{~m}$, $x \approx-6.80 \mathrm{~m}$, and possibly $x \approx-7.60 \mathrm{~m}$ in the pycnocline.

\section{B. Secondary generation, propagation, and decay of internal solitary waves}

To describe the dynamics of internal waves propagating in the pycnocline, the displacement of the isopycnal line $\rho=1040 \mathrm{~kg} / \mathrm{m}^{3}$, initially located at the center of the pycnocline, is studied. The structure of the isopycnal displacement shows no significant change in the vertical direction around the pycnocline (Figure 3(c)), so one can focus on a given isopycne with no loss of information. Owing to the symmetry with respect to $x=0$, we choose as before to focus on leftwards propagating waves in the pycnocline.

The linear horizontal velocity field in Sim9 permits to easily localize the internal wave beams impacts on the pycnocline in Figure 4. Contrary to the primary generation configuration presented in Dossmann, Auclair, and Paci, ${ }^{10}$ the ridge oscillation provokes weak millimetric displacements above the ridge top in the present case. Only at the position of the first internal wave beam impact on the pycnocline $(x \approx-0.25 \mathrm{~m})$ do trains of two steep, narrow troughs develop per forcing period. These troughs are ranked by decreasing depth in a given train (e.g., at $t=17.4 \mathrm{~T}, t=18.4 \mathrm{~T}$, $t=19.4 \mathrm{~T}$ ), and are directed towards the thicker layer, what permits to identify them as secondarily generated internal solitary waves (e.g., Dauxois and Peyrard ${ }^{25}$ ).

The shape of a given ISW2 evolves in the course of its leftwards propagation, as it progressively deepens, before decaying after propagating over approximately $1 \mathrm{~m}$. At $x \approx-0.80 \mathrm{~m}$ and $x \approx-1.90 \mathrm{~m}$, analogous dynamical features are observed after each impact of the initially downgoing internal wave beam: ISW2s are locally generated, then propagate over an approximate distance of $1 \mathrm{~m}$, and eventually decay.

Smoother ISW2s are induced in the pycnocline away from the ridge (e.g., at $x \approx-3.2 \mathrm{~m}$, $x \approx-4.5 \mathrm{~m}$ ). They are caused by the impact of internal wave beams that have been scattered several times, and whose amplitude has weakened due to previous interactions with the pycnocline as well as viscous decay. These ISW2s are, therefore, wider and shallower than in the near field. 

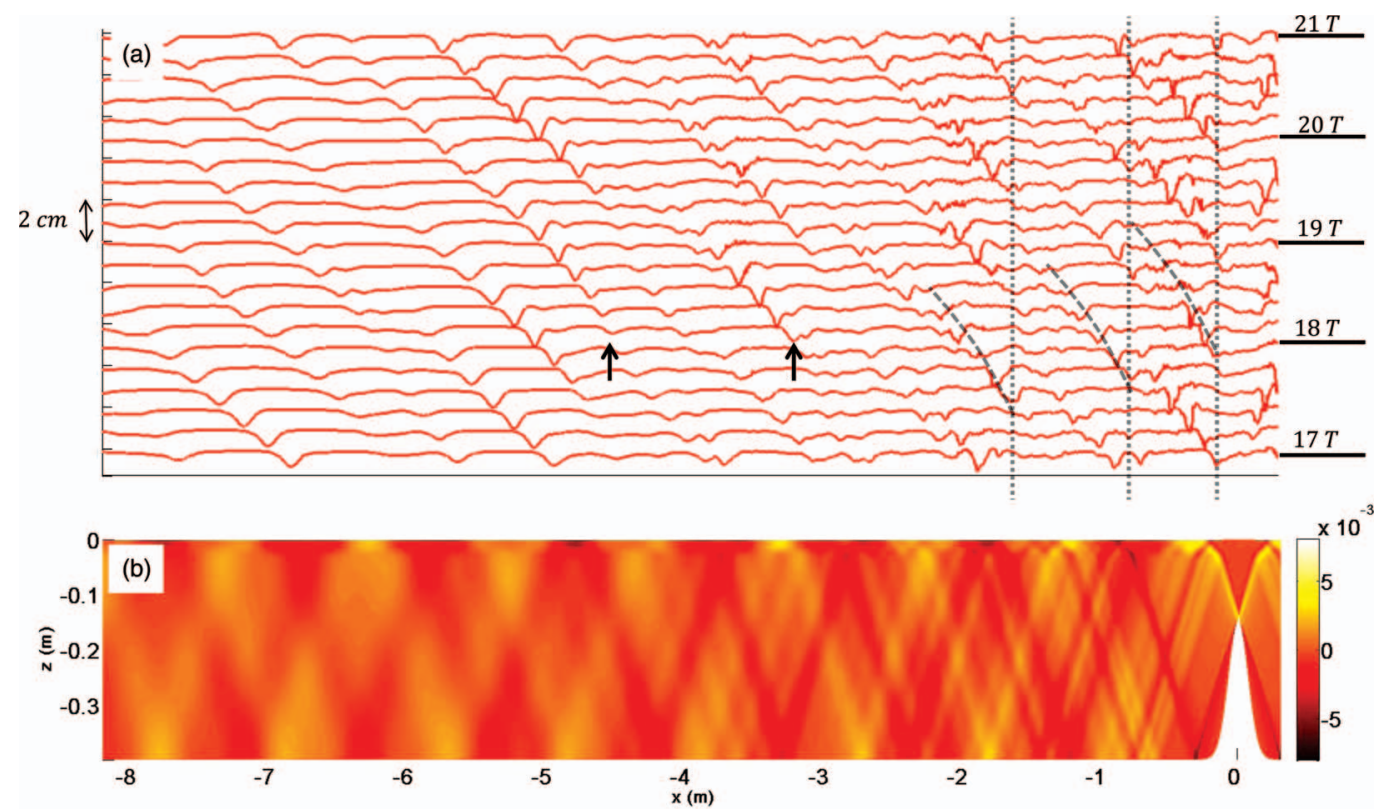

FIG. 4. (a) Displacement $d_{1040}$ of the isopycne $\rho=1040 \mathrm{~kg} / \mathrm{m}^{3}$, for $t=17 \mathrm{~T}, 17.2 \mathrm{~T}, 17.4 \mathrm{~T}, \ldots, 21 \mathrm{~T}$. The vertical step is $2 \mathrm{~cm}$ between two consecutive time steps. Vertical dashed lines indicate the impact region of the upgoing internal wave beam and the two impact regions of the initially downgoing internal wave beam. Tilted dashed line shows the approximate paths followed by the ISW2 generated in these regions. Black arrows indicate the generation of ISW2 from scattered internal wave beams further away from the ridge. (b) Horizontal velocity field $u(\mathrm{~m} / \mathrm{s})$ at $t=22 \mathrm{~T}$, in Sim9.

The observed dynamics of the propagating ISW2s in the pycnocline in Sim10 is similar to the dynamics of ISW2s observed in the ocean (New and Pingree ${ }^{5,11}$ ), or in numerical and physical simulations (Grisouard et al. ${ }^{20}$ Mercier et al. ${ }^{26}$ ).

The space-time diagram shown in Figure 5(a) helps at investigating further into the ISW2s dynamics. To localize nonlinear effects in time and space, the displacement field is projected onto a Morlet wavelet of period T/2 (first harmonic). The result of this projection is shown in Figure 5(b).

Linear and nonlinear waves propagating at different phase speeds are clearly visible in Figure 5(a). The fastest wave, of approximate amplitude $2 \mathrm{~mm}$ propagates at a phase speed of 2.00 $\pm 0.05 \mathrm{~m} / \mathrm{s}$ (blue line in Figure 5(a)), matching the phase speed of surface waves $c_{s}=\sqrt{g H}=2 \mathrm{~m} / \mathrm{s}$. The phase speeds of slower waves are one order of magnitude lower, therefore, one can suppose that they are associated with the emission of normal modes, as tackled in Sec. V. The phase speed of the second fastest wave (red line), of typical amplitude $5 \mathrm{~mm}$, is $0.20 \pm 0.02 \mathrm{~m} / \mathrm{s}$, what corresponds to the theoretical value of the first normal mode phase speed $c_{1}=0.20 \mathrm{~m} / \mathrm{s}$ calculated in Sec. V. These two waves propagate in a quasi-linear manner: only weak harmonics motions, from submillimetric to millimetric amplitudes, are measured.

The generation of ISW2s trains every forcing period is observed on both sides of the ridge. They follow the slope of the third fastest wave (black line) that propagates at $c_{I S W}=0.15 \pm 0.02 \mathrm{~m} / \mathrm{s}$ which is comprised between the phase speeds of the first two normal modes $\left(c_{2}=0.12 \mathrm{~m} / \mathrm{s}\right.$ for the second normal mode). Their decay rate is such that they propagate over approximately $1 \mathrm{~m}$ (black circle in Figure 5(a)). Contrary to the two fastest waves, patches of strong harmonic motions with a typical amplitude of $0.5 \mathrm{~cm}$ characterizing substantial nonlinear effects associated with the propagation of ISW2s. These harmonics motions are trapped in the pycnocline, as the lower stratification is too weak to support their propagation: $2 \omega / N=1.26>1$. Similar harmonic signatures are measured in laboratory experiments describing the interactions of internal wave beams with a pycnocline (Wunsch and Brandt $^{27}$ ) and in previous numerical simulations of the secondary generation process (Grisouard et al. ${ }^{20}$ ).

To evaluate the role played by viscosity on the important energy loss in the pycnocline on a typical length scale of $1 \mathrm{~m}$, the $e$-folding viscous decay length scale of the amplitude $\xi$ is calculated 


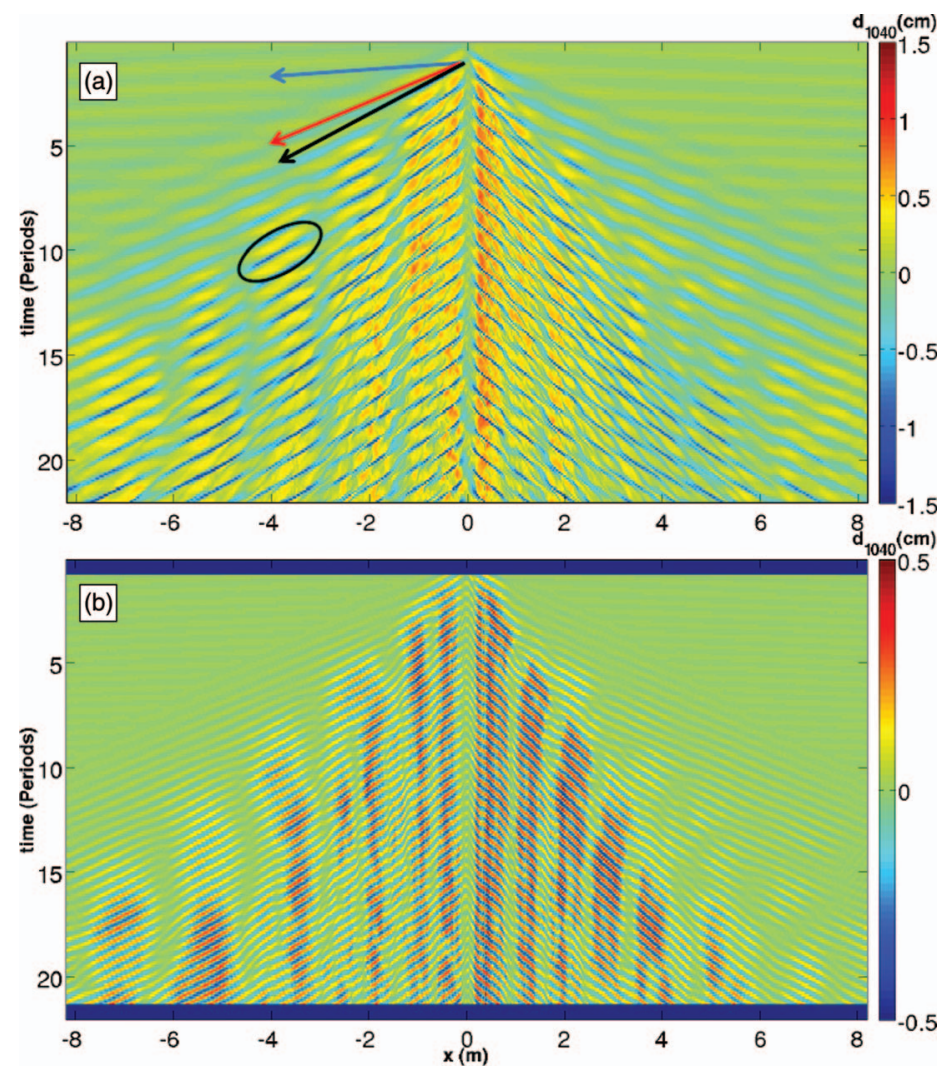

FIG. 5. (a) Space-time diagram of the isopycnal displacement $d_{1040}$, for $\rho=1040 \mathrm{~kg} / \mathrm{m}^{3}$. Arrows indicate the speed of the three fastest waves associated with surface waves $(2.00 \pm 0.05 \mathrm{~m} / \mathrm{s}$, blue), the first normal mode $(0.20 \pm 0.02 \mathrm{~m} / \mathrm{s}$, red), and ISW2 $\left(0.15 \pm 0.02 \mathrm{~m} / \mathrm{s}\right.$, black). (b) Space-time diagram of the isopycnal displacement $d_{1040}$ projected onto a Morlet wavelength with period $\mathrm{T} / 2$.

based on Lighthill, ${ }^{28}$ p. 252 :

$$
\xi=\frac{1}{v}\left(\frac{\hat{\omega}}{\hat{k}}\right)^{3} \frac{\sqrt{\hat{N}^{2}-\hat{\omega}^{2}}}{\hat{N}^{3}}
$$

where $\hat{\omega}$ and $\hat{k}, \hat{N}$ are the values of the typical ISW2 frequency, wavenumber, and the typical Brunt-Väisälä frequency in the pycnocline, respectively. We estimate $\hat{\omega}=3 * 2 \pi / T \approx 1.5 \mathrm{rad} / \mathrm{s}$, $\hat{k}=2 \pi / \lambda_{I S W} \approx 40 \mathrm{rad} / \mathrm{m}$ from Figure 4 , with $\lambda_{I S W} \approx 15 \mathrm{~cm}$ the typical trough width and $\hat{N}$ $\approx 5 \mathrm{rad} / \mathrm{s}$ from the stratification profile (not shown). We find a value $\xi \approx 5 \mathrm{~m}$, five times greater than the observed decay length scale. Hence, viscosity only plays a minor role in the ISW2 amplitude decrease, which is likely led by a downward leaking of energy from ISW2s to internal waves propagating in the lower layer, as described in previous analytical (Gerkema, ${ }^{16}$ Akylas et al. ${ }^{19}$ ) and numerical (Grisouard et al. ${ }^{20}$ ) studies.

In a nutshell, the generation process and the dynamical properties of the propagating troughs show that they are ISW2s. Their propagation speed is close to $c_{2}$, what indicates the dynamics of propagating ISW2s and the second normal mode must be related. The slightly superior value of the ISW2 propagation with respect to the linear normal mode propagation may be caused by the nonlinear advection. In a comparison between in situ observations and a weakly nonlinear approach, New and Pingree ${ }^{29}$ managed to faithfully describe the generation of ISW2s in the Bay of Biscay from the nonlinear evolution of the (single) third normal mode.

In Sec. V, we investigate the relation between the evolution of the normal modes structure with the stratification and the efficiency of the secondary generation process. 


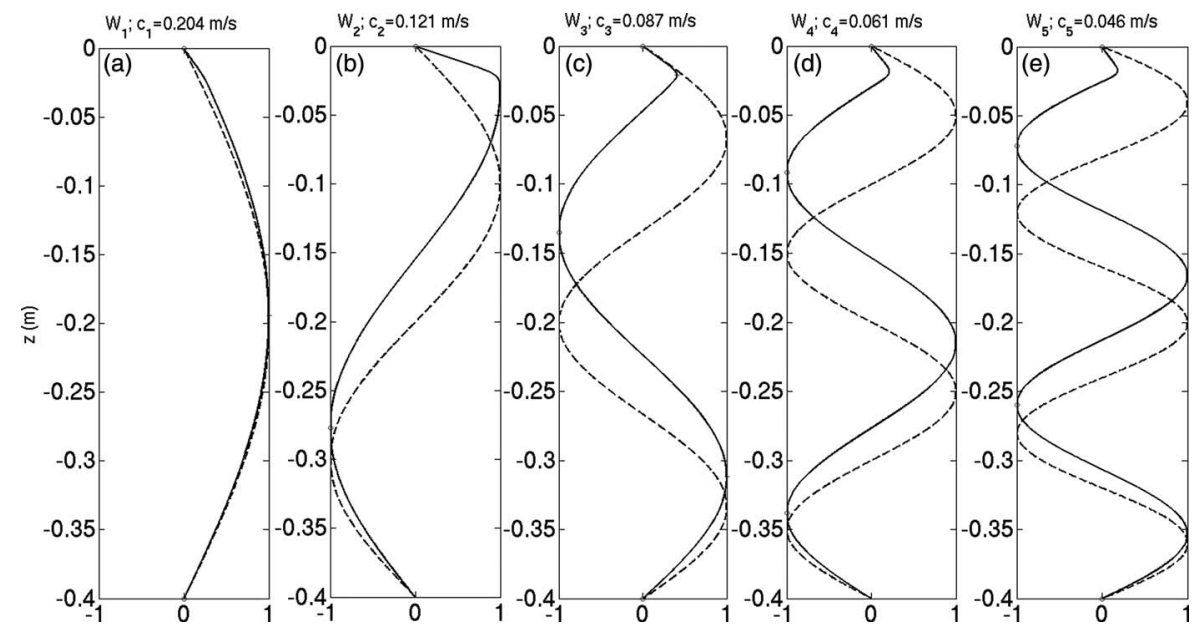

FIG. 6. Plain line: first, second, third, fourth, and fifth normal modes for the vertical velocity and the corresponding phase speeds calculated for the density profile in Figure 1. Dotted black line: First five normal modes for a linear density profile with Brunt-Väisälä frequency $N_{0}$.

\section{NORMAL MODES APPROACH}

\section{A. Principle and phase speeds}

Linear solutions of the inviscid Boussinesq equations for the vertical velocity field are sought in the form

$$
w(x, z, t)=W(z) \exp i(K x-\omega t),
$$

where $W, K$ are the unknown vertical modal structure and wavenumber, respectively, with $K>0$ (respectively, $K<0$ ) for leftwards (respectively, rightwards) propagating waves. $(W, K)$ are solutions of the Sturm-Liouville eigenvalue problem:

$$
\begin{gathered}
\frac{\mathrm{d}^{2} W}{\mathrm{~d} z^{2}}+K^{2} \frac{N^{2}(z)-\omega^{2}}{\omega^{2}} W=0, \\
W(0)=W(-H)=0 .
\end{gathered}
$$

The system of Eqs. (5) and (6) has an infinite, discretized set of solutions $\left(W_{n}, K_{n}\right)$, where $n \in \mathbb{N}^{*}$ is the mode number.

For unidirectional propagating waves, the linear vertical velocity field is retrieved from

$$
w(x, z, t)=\sum_{n=1}^{\infty} a_{n} W_{n}(z) \exp i\left(K_{n} x-\omega t\right) .
$$

The coefficients $\left\{a_{n}\right\}_{n \in \mathbb{N}^{*}}$ are the amplitude of the modes. The phase speed of each mode is defined as $c_{n}=\omega / K_{n}$.

Figure 6 displays the first five normal modes computed from the density profile of Sim9 using Eqs. (5) and (6). In the lower layer, normal modes adopt a quasi-harmonic shape, similar to the case of the linearly stratified fluid, they interfere constructively to form an internal wave beam as observed in Figure 1. In the upper layer instead, the pycnocline modifies strongly the normal modes structure with respect to the linear stratification case, except for the first mode. Consequently, the sum of normal modes after the first reflection leads to a scattered, wider internal wave beam: this is the process of linear scattering.

As ISWs are propagating in a nonlinear manner, they are associated with multiple frequencies. Hence, it is not possible to describe ISWs as a sum of normal modes propagating at a given (tidal) frequency as in the linear case (Eq. (7)). Yet, it is still possible to compare the dynamics of ISW2s and normal modes to understand their relation better. 


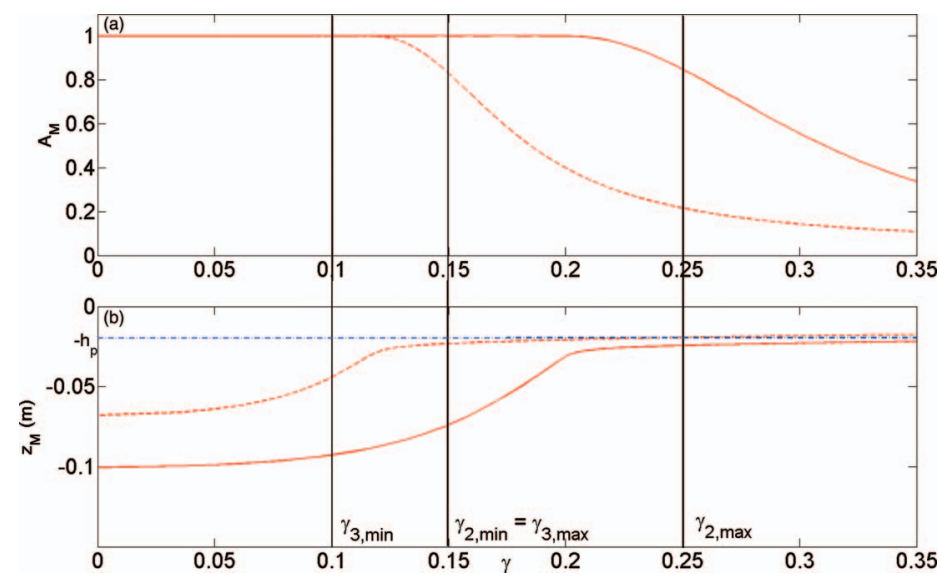

FIG. 7. Solid line (red online): Nondimensional maximal values $A_{M, 2}$ (a) and position $z_{M, 2}$ (b) of the local maximum closest to the pycnocline with respect to $\gamma$, for the second normal mode. Dashed line (red online): same for the third normal mode. The average position of the pycnocline at rest is given by the dashed-dotted line in (b). Plain black lines show the indicative optimal range $\gamma_{2, \min } \leq \gamma \leq \gamma_{2, \max }$ and $\gamma_{3, \min } \leq \gamma \leq \gamma_{3, \max }$ for an efficient secondary generation from the second and third normal modes, respectively.

We observe that the second normal mode propagates at a similar phase speed as the ISW2 in Sim10 and presents a local maximum in amplitude close to the pycnocline rest position. Hence, the second normal mode is likely to generate large vertical displacements in the pycnocline that evolve into ISW2s owing to the balance between nonlinear and nonhydrostatic effects.

\section{B. Evolution of the normal modes structure}

The parameter $\gamma=c^{*} /\left(N_{0} H\right)$ is introduced by Gerkema ${ }^{16}$ to quantify the pycnocline strength. The case $\gamma \rightarrow+\infty$ corresponds to the limit of a two-layer fluid with a density jump $\Delta \rho$ and no stratification in the bottom layer, and the case $\gamma \rightarrow 0$ to the limit of a linearly stratified fluid. An intermediate value of $\gamma$ corresponds to a fluid composed of a pycnocline lying over a stratified lower layer. Internal waves present an intertwined behavior in that case as they can propagate horizontally in the pycnocline as well as an inclined internal wave beam in the lower layer. Gerkema ${ }^{16,17}$ shows that the secondary generation is favored from the nonlinear evolution of the third normal mode for a moderate pycnocline $(\gamma \approx 0.1)$.

We propose to extent these previous studies by examining the evolution of the structure of a given normal mode with respect to $\gamma$. While these studies, along with New and Pingree, ${ }^{29}$ showed that ISW2s are likely to emerge from the third normal mode in the Bay of Biscay, we choose first to focus on the second normal mode that appears to be the best candidate to degenerate into an ISW2 in the present configuration (Sec. V A).

To do so, the vertical position $z_{M, 2}$ and nondimensional amplitude $A_{M, 2}$ of the local maximum closest to the pycnocline are measured for varying $\gamma$, as shown in Figures 7(a) and 7(b).

The closest local maximum to the pycnocline varies in amplitude and position with $\gamma$. For a relatively weak pycnocline $\left(\gamma \leq \gamma_{2, \min }\right.$ with $\left.\gamma_{2, \min } \approx 0.15\right), A_{M, 2} \approx 1$ and $z_{M, 2} \approx-10 \mathrm{~cm}$, while the pycnocline is at $z=-h_{p}=-2 \mathrm{~cm}$. Hence, if the second normal mode is excited in this configuration, the strongest vertical displacements occur too deep in the fluid to generate important vertical displacements in the pycnocline. In the opposite limit of a strong pycnocline $\left(\gamma \geq \gamma_{2, \max }\right.$ with $\gamma_{2, \min } \approx 0.25$ ), the local maximum is located in the pycnocline, but its amplitude decreases fast: the pycnocline becomes too "rigid" to support important displacements for the second normal mode.

For intermediate pycnocline strength $\left(\gamma_{2, \min } \leq \gamma \leq \gamma_{2, \max }\right)$, the maximal amplitude remains close to 1 while the maximum is relatively close to the pycnocline $\left(z_{M, 2}=-0.03 \mathrm{~m}\right.$ for $\left.\gamma \approx 0.20\right)$.

Hence, for a value of $\gamma$ in the range $\left[\gamma_{2, \min }, \gamma_{2, \max }\right]$ : 
- $A_{M, 2}$ is close to 1 , causing potential large vertical displacements.

- $z_{M, 2} \approx-h_{p}$, so the maximal vertical displacements are located in the pycnocline.

Nonlinear effects leading to the degeneration of the second normal mode into an ISW2 are likeliest to occur when these two conditions are reached. A similar behavior of the normal mode structure is observed at least for the first ten modes. The range of $\gamma$ values for which the previous compromise is met decreases with increasing mode number. The example of the third normal mode is also shown in Figures 7(a) and 7(b). In that case, the optimal structure for the normal mode is obtained in the range $0.10 \leq \gamma \leq 0.15$, in accordance with Gerkema. ${ }^{16}$ Consequently, the stronger the pycnocline, the lower the number of the normal mode that may evolve into an ISW2. In the limit of $N_{0}=0(\gamma \mapsto \infty)$, ISW1s are generated from the disintegration of the first normal mode.

This interpretation introduces a new general compromise, quantified by $\gamma$ and valid for all normal modes, between the value of the (nondimensional) local maximum close to the pycnocline, and its relative distance from the pycnocline, to describe the efficiency of ISW2 generation.

To induce large vertical displacements in the pycnocline, a given normal mode $n$ that respects the previous compromise must be efficiently forced. This supplementary condition is discussed below.

\section{Role of the topography}

In the configuration of Sim10, the oscillation of the topography leads to the emission of normal modes whose amplitudes $\left\{a_{n}\right\}_{n \in \mathbb{N}^{*}}$ are imposed by the oscillation of the topography of given shape. In oceanic configurations, the normal mode amplitudes are imposed by the barotropic tide/topography interaction. The shape of the topography plays a key role in the secondary generation process, since it controls the energy input into the normal mode that matches the compromise introduced in Subsection V B. Providing such a mode is efficiently forced, ISW2s can emerge from the nonlinear evolution of this mode in the pycnocline.

Note that in the ocean, the amplitude $a_{n}$ usually decreases with the mode number $n$. Therefore, one can expect that independently of the pycnocline strength, low order normal modes are likelier to evolve into ISW2 than high order normal modes.

\section{DISCUSSION}

To improve our understanding of the secondary generation process, we propose to relate previous independent approaches with the present work. We first recall the selection criteria described in the studies of Akylas et al. ${ }^{19}$ and Grisouard et al. ${ }^{20}$

\section{A. Matching the internal wave beam and the interfacial wave wavelengths, or phase speeds}

The study of Akylas et al. ${ }^{19}$ relies on a weakly nonlinear nonhydrostatic model with a pycnocline with zero thickness. Contrary to Gerkema, ${ }^{16}$ a lower layer of infinite depth as well as a prescribed profile for the internal wave beam structure of maximum amplitude for the vertical velocity $A_{w}$ and wavelength $\lambda_{0}$ is adopted.

Akylas et al. ${ }^{19}$ first address the issue of the generation of a linear wave in the pycnocline due to an impinging internal wave beam. The shape of the induced interfacial displacement near the internal wave beam impact is studied with respect to the internal wave beam amplitude and wavelength.

It is shown that the parameter $\alpha=N_{0} \lambda_{0} / c^{*}$ controls the efficiency of the energy transfer between internal wave beams propagating in the lower layer and an interfacial wave in the pycnocline. The authors come to the conclusion that a moderate pycnocline, associated with $\alpha=O(1)$, leads to large vertical displacements in the pycnocline from which ISW2s may arise. The spatial resonance process here at play is the matching between the horizontal wavelength of the internal wave beam $\lambda_{0} / \sin (\theta)$ on one hand and the interfacial wavelength $2 \pi c^{*} /\left(N_{0} \sin \theta\right)$ on the other hand. It can be equivalently described by the matching of the internal wave beam phase speed $c_{\phi, x}$ and the horizontal interfacial wave phase speed $c^{*}$, as the two waves oscillate at $\omega$ before nonlinear effects come into play. 
TABLE II. Characteristic phase speed ratio describing the efficiency of ISW2 generation. $c_{1, l}=N_{0} H$ is the phase speed of the first normal mode for a linear stratification $N_{0}$.

\begin{tabular}{|c|c|c|}
\hline Authors & Fluid properties & Phase speeds ratio \\
\hline Gerkema $^{16}$ & Zero thickness pycnocline, finite depth & $c^{*} / c_{1, l}$ \\
\hline Akylas et al. ${ }^{19}$ & Zero thickness pycnocline, infinite depth & $c^{*} / c_{\phi, x}$ \\
\hline Grisouard et al. ${ }^{20}$ & Finite thickness pycnocline, finite depth & $c_{n} / c_{\phi, x}$ \\
\hline Present work & Finite thickness pycnocline, finite depth & $c_{n} / c_{I S W}$ \\
\hline
\end{tabular}

In Sim10, the parameter $\alpha$ is of the order of magnitude of 1 , corresponding to the spatial resonance condition proposed by Akylas et al. ${ }^{19}$

\section{B. Matching the internal wave beam and normal mode phase speeds}

Grisouard et al..$^{20}$ adopt a more realistic stratification by studying the effects of a finite thickness pycnocline. They design an experiment with an analytical internal wave beam and a stratification defined by Eq. (1). Adapting the physical parameters so that the two conditions $\gamma \approx 0.1$ and $\alpha=0(1)$ are satisfied, they describe the dynamics of the secondary generation process. The authors argue that the generation of one mode- $n$ ISW2s train occurs when the ratio $c_{n} / c_{\phi, x} \approx 1$, where $c_{n}$ is the phase speed of a normal mode $n$ trapped in the pycnocline, and $c_{\phi, x}$ is the horizontal phase speed of the internal wave beam.

In Sim10, mode-1 ISW2s, consisting in a single depression in the pycnocline, are observed. The horizontal wavelength of the beam, before impinging on the pycnocline, equals $6 \pm 1 \mathrm{~cm}$, the uncertainty being due to the small scale structures present in the vicinity of the beam. It corresponds to a phase speed $c_{\phi, x}=1.2 \pm 0.2 \mathrm{~cm} / \mathrm{s}$. For the first normal mode trapped in the pycnocline, associated with a frequency $\Omega>N_{0}$, one find $c_{1} / c_{\phi, x}=8.0 \pm 1.2$, indicating that the selection criterion introduced by Grisouard et al. ${ }^{20}$ is not at play in the present configuration.

\section{Relating the approaches}

The different physical interpretations leading to the same conclusion that ISW2s are efficiently generated in a moderate pycnocline regime $(\gamma \approx 0.1$ or $\alpha \approx 1)$ appear weakly related (Gerkema, ${ }^{16,17}$ Akylas et al. ${ }^{19}$ Grisouard et al. ${ }^{20}$ ).

Interestingly, the studies of Gerkema, ${ }^{16}$ Akylas et al. ${ }^{19}$ Grisouard et al.${ }^{20}$ as well as the present work all involve a different phase speed matching associated with an efficient ISW2 generation, as summarized in Table II.

Focussing on the trigger of the secondary generation is a step to propose a unified description of this process. On the one hand, it is observed that one ISW2s train is generated every tidal period at the location of the internal wave beam impact on the pycnocline. On the other hand, several studies, including the present one, argue that the ISW2 generation is triggered by the nonlinear evolution of one given normal mode (the second mode in the present work). Therefore, one can infer that the nonlinear evolution of a given normal mode must occur at the location of the beam impact. These two mechanisms are in fact related: in a modal view, the beam/pycnocline interaction is caused by normal modes interfering constructively in this region, thereby inducing large vertical displacements. The main contributor to these large displacements is the normal mode 2 , which can degenerate into an ISW2 when nonlinear effects come into play. Away from the beam impact, the large amplitude of the second normal mode at the pycnocline is cancelled by the other normal modes. Consequently, the vertical displacements are too weak for nonlinear effects to develop.

Finally, one can wonder whether the wavelength matching criterion in Akylas et al.' ${ }^{19}$ approach and the compromise of the normal mode structure described in the present work are fully equivalent. Concentrating on the role of the topography can help at providing more insights regarding this 
TABLE III. Selection criteria for ISW generation for the primary generation and in the normal modes and in the internal wave beam/pycnocline approaches of the secondary generation. Solid and dashed lines indicate the parameters controlled by the stratification and the ridge, respectively. $\mathrm{T}$ is the period of the barotropic tide. $A_{w, r}$ is the amplitude of the vertical displacement induced by the barotropic tide/ridge interaction. Amplitudes are nondimensionalized by the mixed layer depth $h_{1} . \lambda_{r}$ is the characteristic wavelength of the ridge and $c^{*}$ is the interfacial propagation speed in the case of a two-layer fluid with constant densities in the two layers. Other terms are introduced in Secs. II-VI.

\begin{tabular}{|c|c|c|c|}
\hline \multicolumn{2}{|l|}{ Generation mechanism } & \multirow{2}{*}{$\begin{array}{c}\text { Physical parameters: stratification/ridge } \\
\qquad \underline{A_{w, r}, \lambda_{r}, \underline{c^{*}}, h_{1}}\end{array}$} & \multirow{2}{*}{$\frac{\text { Criterion ISW generation }}{c^{*} \times T / \lambda_{r} \approx 1, \text { large } A_{w, r} / h_{1}}$} \\
\hline Primary generation & & & \\
\hline Secondary generation & Normal modes & $\left\{a_{n}\right\}_{n \in \mathbb{N}^{*}}, \gamma, h_{1},\left\{\left[\gamma_{n, \min }, \gamma_{n, \max }\right]\right\}_{n \in \mathbb{N}^{*}}$ & $\gamma \in\left[\gamma_{n, \min }, \gamma_{n, \max }\right]$, large $a_{n} / h_{1}$ \\
\hline & $\begin{array}{l}\text { Internal wave } \\
\text { beam/pycnocline }\end{array}$ & $\underline{A_{w}, \alpha,}, \underline{h_{1}}$ & $\alpha \approx 1$, large $A_{w} / h_{1}$ \\
\hline
\end{tabular}

question. In fact, the structure of a propagating internal wave beam in the lower layer is equivalently described by the set $\left\{a_{n}, W_{n}, c_{n}\right\}_{n \in \mathbb{N}^{*}}$ in a normal mode approach or $\left(A_{w}, \lambda_{0}, \theta\right)$, in the internal wave beam approach. These two related physical quantities are involved in the selection criteria proposed in the previous studies, and have always been studied separately. Therefore, future works may interestingly relate their respective evolutions when varying the topography shape. Such study would help at providing a unified description of the secondary generation mechanism.

\section{CONCLUSION}

To our knowledge, this study shows the first direct numerical simulations of the secondary generation of ISWs by topographic internal wave beams. In the linear regime, the generation of smooth internal wave beams in the lower, linearly stratified layer, and the subsequent linear scattering at the pycnocline are observed. Increasing the forcing amplitude 20 times leads to the generation of wider and more turbulent internal wave beams in the bottom layer inducing sharp, localized troughs when impinging on the pycnocline. These troughs propagate over approximately $1 \mathrm{~m}$ in the pycnocline, before undergoing an amplitude decrease due to a downward leaking of energy. The overall dynamics of the interfacial waves is characteristic of ISW2 propagation, described in various studies (Grisouard et al. ${ }^{20}$ and references therein).

To help at clarifying the secondary generation process, we focus on the evolution of the spatial structure of a given normal mode with respect to $\gamma$. We describe at first the second normal mode which propagates at a similar phase speed as the observed ISW2. The largest vertical displacements are induced in the pycnocline by this mode when a compromise between the position and the amplitude of the closest maximum to the pycnocline appears. This general compromise, valid for every mode, is reached for a range of pycnocline strength whose mean value decreases with the normal mode number. The topography hence appears to play a prominent, although indirect, role in the secondary generation process, since it controls the amplitude $a_{n}$ of the normal mode from which ISW2 can arise. We propose ideas to unify the normal modes and the internal wave beams/pycnocline approaches to the secondary generation process.

Analogies can be made with the selection criterion in the primary generation configuration extracted by Dossmann, Auclair, and Paci. ${ }^{10}$ Large amplitude ISW1s are generated when the interfacial wavelength $c^{*} \times T$ matches the characteristic wavelength of the topography $\lambda_{r}$, and for a sufficient barotropic forcing.

In these two regimes, the efficiency of ISWs generation can be summarized by two nondimensional parameters: one parameter describing a selection criterion (matching between horizontal wavelengths or optimal vertical structure for a normal mode), and one parameter quantifying the vertical displacements. Table III summarizes these parameters.

For an oceanic purpose, this study along with Dossmann, Auclair, and Paci ${ }^{10}$ provides tools to understand better the mechanisms involved in the tide-topography-ISW interaction, in the primary and secondary generation processes. To get closer to realistic configurations, future studies could 
focus on the effect of a shear flow in the two generation processes. The interaction between interfacial waves emitted over the topography and ISW2s that may control the distance between consecutive ISW2s in the ocean is another important physical process that requires further description (Grisouard and Staquet $^{21}$ ).

\section{ACKNOWLEDGMENTS}

This work has been supported by LEFE-IDAO Programme "ondes et marées internes dans l'océan" (LEFE-IDAO-07/2) and ANR "PIWO” Contract No. (ANR-08-BLAN-0113). Y. Dossmann's Ph.D. thesis was funded by a MNERT scholarship. We thank the POC team (LA, UMR 5560, Paul Sabatier University and CNRS and LEGOS, UMR 5566, CNES, CNRS, IRD Paul Sabatier University) for their kind support. We thank T. Gerkema and the two anonymous reviewers for helpful discussions. Numerical experiments were performed on the French supercomputer centers CALMIP, through projects p1052 and p1054, and CINES, through project x2013016618 and on the Laboratoire d'Aérologie cluster (a great thanks to the LA computing team).

${ }^{1}$ V. C. Tsai, H. Kanamori, and J. Artru, “The morning glory wave of Southern California,” J. Geophys. Res. 109, B02307, doi:10.1029/2003JB002596 (2004).

2 J. W. Rottman and R. Grimshaw, Internal Solitary Waves (Kluwer, 2001), pp. 61-88.

${ }^{3}$ J. Apel, J. Holbrook, A. Liu, and J. Tsai, “The Sulu Sea internal soliton experiment,” J. Phys. Oceanogr. 15, 1625-1651 (1985).

${ }^{4}$ D. Halpern, “Semidiurnal internal tides in Massachusetts Bay,” J. Geophys. Res. 76, 6573-6584, doi:10.1029/JC076i027p06573 (1971).

${ }^{5}$ A. L. New and R. D. Pingree, "Large-amplitude internal soliton packets in the central Bay of Biscay," Deep-Sea Res., Part A 37, 513-524 (1990).

${ }^{6}$ K. H. Helfrich and W. K. Melville, “Long nonlinear internal waves,” Annu. Rev. Fluid Mech. 38, 395-425 (2006).

${ }^{7}$ L. S. Kantha and C. A. Clayson, "An improved mixed layer model for geophysical applications," J. Geophys. Res. 99, 25235-25266, doi:10.1029/94JC02257 (1994).

${ }^{8}$ Z. Lai, C. Chen, R. C. Beardsley, B. Rothschild, and R. Tian, "Impact of high-frequency nonlinear internal waves on plankton dynamics in Massachusetts Bay," J. Mar. Res. 68, 259-281 (2010).

${ }^{9}$ R. D. Pingree and G. T. Mardell, "Solitary internal waves in the Celtic Sea," Prog. Oceanogr. 14, 431-441 (1985).

${ }^{10}$ Y. Dossmann, F. Auclair, and A. Paci, "Topographically induced internal solitary waves in a pycnocline: Primary generation and topographic control," Phys. Fluids 25, 066601 (2013).

${ }^{11}$ A. L. New and R. D. Pingree, "Local generation of internal soliton packets in the central Bay of Biscay," Deep-Sea Res., Part A 39, 1521-1534 (1992).

${ }^{12}$ A. L. New and J. C. B. Da Silva, "Remote-sensing evidence for the local generation of internal soliton packets in the central Bay of Biscay," Deep-Sea Res. 49, 915-934 (2002).

${ }^{13}$ A. Azevedo, J. C. B. da Silva, and A. L. New, "On the generation and propagation of internal solitary waves in the southern Bay of Biscay,” Deep-Sea Res., Part I 53, 927-941 (2006).

${ }^{14}$ D. I. Delisi and I. Orlanski, "On the role of density jumps in the reflexion and breaking of internal gravity waves," J. Fluid Mech. 69, 445-464 (1975).

${ }^{15}$ S. A. Thorpe, "Nonlinear reflection of internal waves at a density discontinuity at the base of the mixed layer," J. Phys. Oceanogr. 28, 1853-1860 (1998).

${ }^{16}$ T. Gerkema, "Internal and interfacial tides: beam scattering and local generation of solitary waves," J. Mar. Res. 59, 227-255 (2001).

${ }^{17}$ T. Gerkema, "Development of internal solitary waves in various thermocline regimes—a multi-modal approach," Nonlin. Processes Geophys. 10, 397-405 (2003).

${ }^{18}$ R. Maugé and T. Gerkema, "Generation of weakly nonlinear nonhydrostatic internal tides over large topography: a multi-modal approach,” Nonlinear Processes Geophys. 15, 233-244 (2008).

${ }^{19}$ T. R. Akylas, R. H. J. Grimshaw, S. R. Clarke, and A. Tabaei, "Reflecting tidal wave beams and local generation of solitary waves in the ocean thermocline," J. Fluid Mech. 593, 297-313 (2007).

${ }^{20}$ N. Grisouard, C. Staquet, T. Gerkema et al., "Generation of internal solitary waves in a pycnocline by an internal wave beam: a numerical study," J. Fluid Mech. 676, 491 (2011).

${ }^{21}$ N. Grisouard and C. Staquet, "Numerical simulations of the local generation of internal solitary waves in the Bay of Biscay," Nonlinear Processes Geophys. 17, 575-584 (2010).

${ }^{22}$ F. Auclair, C. Estournel, J. W. Floor, M. Herrmann, C. Nguyen, and P. Marsaleix, "A non-hydrostatic algorithm for free-surface ocean modelling," Ocean Modell. 36, 49-70 (2011).

${ }^{23}$ Y. Dossmann, A. Paci, F. Auclair, and J. W. Floor, "Simultaneous velocity and density measurements for an energy-based approach to internal waves generated over a ridge," Exp. Fluids 51, 1013-1028 (2011).

${ }^{24}$ M. Mathur and T. Peacock, "Internal wave beam propagation in non-uniform stratifications," J. Fluid Mech. 639, 133-152 (2009).

25 T. Dauxois and M. Peyrard, Physics of Solitons (Cambridge University Press, New York, 2006). 
${ }^{26}$ M. J. Mercier, M. Mathur, L. Gostiaux, T. Gerkema, J. M. Magalhaes, J. C. B. da Silva, and T. Dauxois, "Soliton generation by internal tidal beams impinging on a pycnocline: laboratory experiments," J. Fluid Mech. 704, 37-60 (2012).

${ }^{27}$ S. Wunsch and A. Brandt, "Laboratory experiments in internal wave interactions with a pycnocline," Exp. Fluids 53, 1663-1679 (2012).

${ }^{28}$ J. Lighthill, Waves in Fluids (Cambridge University Press, 1978).

${ }^{29}$ A. L. New and R. Pingree, "An intercomparison of internal solitary waves in the Bay of Biscay and resulting from Korteweg-de Vries-type theory,” Prog. Oceanogr. 45, 1-38 (2000). 